\title{
Historia de las mujeres, género y musicología:
}

\author{
herramientas para analizar el disco \\ mujer/Mujer (1984) de Chany Suárez
}

Tomás Agustín Mariani

[Universidad Nacional de Quilmes - CONICET]

Resumen En la primera parte de este artículo presentamos algunos hitos importantes del devenir de la historia de las mujeres en la historiografía tomando como eje a la categoría de género. Nos detenemos en los casos de Estados Unidos, apoyándonos en la perspectiva de Joan Scott (2003), y de Argentina, apoyándonos en la perspectiva de Dora Barrancos (2005). Posteriormente, articulamos la categoría de género con el devenir de la historia de las mujeres en el ámbito de la musicología. En la segunda parte de este artículo presentamos un análisis del disco mujer/MUJER (1984) de Chany Suárez retomando herramientas presentadas en la primera parte.

Este trabajo se inscribe dentro de una investigación más amplia, que abarca referentes emergentes del folklore argentino entre el fin de la dictadura (1982-1983) y el comienzo de la democracia. En ese sentido, nuestra propuesta de análisis considera a este disco como un dispositivo de enunciación, en línea con nuestros anteriores trabajos (Mariani, 2019a; 2019b y 2020).

Palabras clave: folklore -

Chany Suárez · género
Summary In the first part of this article, we present some important milestones in the development of women's history in historiography, taking as an axis the category of gender. We look at the cases of the United States, from the perspective of Joan Scott (2003), and of Argentina, from the perspective of Dora Barrancos (2005). Subsequently, we articulate the category of gender with the evolution of women's history in the field of musicology. In the second part of this article, we present an analysis of the record mujer/MUJER (1984) by Chany Suárez, taking up tools presented in the first part.

This work is part of a broader investigation, covering emerging referents in Argentine folklore between the end of the dictatorship (19821983) and the beginning of democracy. In this sense, our proposal of analysis considers this record as a device of enunciation, in line with our previous works (Mariani, 2019a; 2019b and 2020).

Keywords: folklore ·

Chany Suárez $\cdot$ gender 


\section{$1 \cdot$ InTRODUCCIón}

En los últimos años han crecido en el ámbito de la musicología argentina y latinoamericana los trabajos que retoman discusiones feministas. Se trata de un proceso que sin dudas se ve alimentado por la renovada presencia pública de dichas discusiones. Como parte de ese proceso, en este artículo relevamos algunos aspectos de la historia de las mujeres y la categoría de género en la historiografía y en la musicología para luego analizar desde esa perspectiva un disco de Chany Suárez.

El trabajo que aquí presentamos se inscribe dentro de una investigación más amplia, que abarca referentes emergentes del folklore argentino entre el fin de la dictadura (1982-1983) y el comienzo de la democracia. Chany Suárez se encuentra entre las referentes que estudiamos. Continuando la línea de nuestros anteriores trabajos (Mariani, 20I9a; 2019b y 2020), proponemos un análisis de su disco mujer/MUJER (1984) como un dispositivo de enunciación ${ }^{1}$. En este caso, tomando como punto de partida de nuestro análisis que desde el propio título observamos que se presenta a una enunciadora mujer.

\section{2 - Historia de las MUjeres, géNero y MUSicología}

\section{$2.1 \cdot$ Aspectos de la Historia de las mujeres y la categoría de género}

La historiadora estadounidense Joan Scott (2003), en un trabajo de revisión crítica, afirma que la Historia de las mujeres se consolida como campo de estudios desde la década de 1970, y llega a convertirse en un movimiento internacional (más allá de que ella se centra en la experiencia de Estados Unidos). Asimismo, la autora, considera que la política

1 En nuestros trabajos sobre folklore en los años ochenta, incluido el abordaje de este disco de Chany Suárez, retomamos el modelo socio-discursivo desarrollado por Claudio Díaz (2009). En esa perspectiva los discos son considerados como dispositivos de enunciación que construyen enunciadoras/es y enunciatarios/as (que no deben confundirse con el/la artista "real" y los/as receptores/escuchas "reales"). 
feminista de los años sesenta fue el punto de partida para la consolidación de ese campo de estudios.

La historiadora argentina Andrea Andújar (20I2), también en un trabajo de revisión crítica, agrega que aquella política feminista (que llegó a consolidarse internacionalmente como «Movimiento de Liberación de la Mujer» en países como Estados Unidos, Canadá, Gran Bretaña, Francia e Italia), se dio en simultaneidad con luchas contra la explotación de clases, el imperialismo o el racismo. Desde allí las historiadoras feministas se encaminaron hacia la construcción de una Historia de las mujeres.

Como indica Andújar (2012), se procuró restituir a las mujeres en la historia y a la vez proporcionar materiales para luchar contra las desigualdades y la subordinación. Los primeros estudios abordaron mujeres que aportaron a los grandes procesos de cambio social ejerciendo roles semejantes a los de los hombres de la historia tradicional. A partir de esos trabajos se visibilizó la escasez y la parcialidad masculina de las fuentes tradicionales (las oficiales, estatales o públicas) para este tipo de temas y la necesidad de replantear las preguntas. Dónde estaban, qué habían hecho y cómo se consideraba socialmente a las mujeres fueron las nuevas líneas de investigación, que a su vez incorporaron nuevas fuentes: memorias, diarios personales, periódicos, literatura. Andújar afirma que esa historiografía logró visibilizar diversas formas de opresión, así como las reacciones ante las mismas, pero no respondía al por qué de la ausencia de las mujeres en los relatos históricos. Además, contenía el riesgo de construir una historia paralela desvinculada del desarrollo económico, político y social. Como respuesta a esto surgieron trabajos (destacándose el de Joan Kelly ${ }^{2}$ ) que confrontaron las experiencias de mujeres con las de varones en un mismo período. Se logró así demostrar un problema central de la historiografía: el varón había sido el eje explicativo del desarrollo de las sociedades. A este modo de construcción de relatos del pasado se lo empezó a nombrar como androcéntrico. El desafío era, como describe Andújar, elaborar una narrativa histórica que contuviera las experiencias de ambos sexos.

2 Kelly, Joan (1990 [1977]): “¿Tuvieron las Mujeres Renacimiento?», en Amelong, J. y Nash, M. (comps.): Historia y género: las mujeres en la Europa moderna y contemporánea. Valencia: Alfonso el Magnánimo. 
El caso estadounidense, abordado por Joan Scott (2003), es claro respecto de los conflictos que se presentaron en el devenir del movimiento de la Historia de las mujeres. Scott considera de modo relacional el carácter dinámico del feminismo, de la disciplina historia y de la Historia de las mujeres (aunque evitando pensarlas como reflejos unas de otras).

Durante la década de 1960, en Estados Unidos, a raíz de la relación entre el feminismo y la lucha por los Derechos Civiles, así como la instalación de una retórica de la igualdad por parte del gobierno demócrata, se dio un proceso de disputa de espacios y una lucha contra la discriminación por género. En esa etapa se lograron avances paulatinos en materia institucional, tanto en el estado como en lo laboral, en las organizaciones propias, en los partidos políticos y en las universidades. A partir de esto, las feministas del mundo académico avanzaron en la disputa de espacios, reconocimientos y derechos, conformando en ese proceso una nueva identidad colectiva de las mujeres de este ámbito. En particular, en la Asociación Histórica Americana (AHA) la disputa tuvo, como reacción de los referentes establecidos, la acusación de falta de profesionalismo entre las mujeres que reclamaban, agregando además que politizaban la institución. Scott afirma que las respuestas a esa acusación apuntaron a poner de manifiesto que «las profesiones son organizaciones políticas (en los múltiples sentidos de la palabra "política")» (2003: 66). Las luchas al interior de las asociaciones profesionales (en particular la de historia) no solo pusieron en tensión la definición de las reglas del mundo académico sino también las jerarquías y asimetrías de la propia profesión.

Uno de los conflictos que Scott marca como centrales para las historiadoras feministas estaba entre aportar «un complemento inofensivo de la historia instituida y una sustitución radical de la misma» (2003: 68). El problema radicaba en construir una historia de las mujeres que complemente la historia ya escrita, la del sujeto universal hombre y blanco, o proponer una ruptura con ésta en función de una historia relacional que expusiera las jerarquías implícitas en los relatos tradicionales.

Frente a las acusaciones por parte de historiadores «tradicionales", Scott sugiere que muchas historiadoras evitaron las discusiones epistemológicas y metodológicas más radicales, y se centraron en «el papel 
de la mujer como materia histórica adicional» (2003: 73), a modo de recurso táctico.

Es en esta etapa que se asociaron al campo de la historia social. Lograron que se le otorgue a las mujeres, junto con otros grupos, "el rango de sujetos históricos» (2003: 74) en la disciplina historia, aunque sin discutir la «objetividad» de las historias tradicionales. Para esto definieron a las mujeres como "una categoría social fija» (2003: 74). Así, mujeres por un lado y hombres por otro, se definían como grupos de interés homogéneo. En algunos casos se trabajó sobre la similitud y, en otros, sobre la diferencia entre actividades de hombres y mujeres. A su vez, se acentuó menos la victimización de las mujeres que la afirmación de la "cultura femenina», procurando dar pruebas de su «capacidad para hacer historia» (2003: 75). Scott resume que «la historia de las mujeres logró cierta legitimidad como tarea histórica al afirmar la naturaleza diversa, la experiencia aparte de las mujeres, o lo que es lo mismo, cuando consolidó la identidad colectiva de las mujeres» (2003: 76).

Hacia fines de los años setenta, tanto historiadoras como movimientos políticos feministas, problematizaron el acento en la «diferencia». De esa discusión emerge el concepto de género como herramienta teórica ${ }^{3}$ para pensar la diferencia sexual, con acento social (evitando el esencialismo biológico) y pensando de modo relacional estas diferencias que, a su vez, pueden cambiar en el tiempo y en distintas sociedades. Además, tal como relata Scott, la categoría de género permitió analizar las diferencias dentro de las diferencias cruzando otras categorías como etnia, clase o región.

Esto puso en cuestión la idea de las mujeres como una categoría fija, como un grupo de intereses homogéneos. A su vez, puso en crisis la propia idea de una Historia de las mujeres. Una de las vías por las que se pensó este problema, con herramientas del postestructuralismo, fue pasar «de suponer una identidad preexistente en las 'mujeres' a investigar los procesos de su construcción; de atribuir un significado inherente

3 En un trabajo muy difundido y valorado Scott (1990) sistematiza reflexiones sobre la categoría género, considerando aspectos simbólicos, normativos, políticos y subjetivos. 
a categorías como "hombre" y "mujer" a analizar cómo se afianza su significado» (Scott, 2003: 80).

Estos cuestionamientos desde el postestructuralismo fueron discutidos en los movimientos feministas, considerando algunos sectores que se trataba de una "teoría» alejada de la práctica "política». Frente a esta polarización, Scott pregona que es posible una articulación entre teoría y política.

Finalmente, Scott hace una revisión de lo ocurrido durante la década de 1990. Afirma que en esa etapa hay una avalancha de historiografía sobre las mujeres con un gran éxito del enfoque de género. La historia de las mujeres no ocupó un lugar central en el campo de la historia, aunque sí logró aportar a la pluralidad de agentes y de historiadores, a la vez que debilitar la hegemonía de la historia tradicional. Es así que la historia de las mujeres logró un espacio de poder dentro de la disciplina. Scott ve las tensiones, en esta etapa, al interior del campo de la historia de las mujeres: historia frente a teoría, universalismo frente a particularismo e incluso mujeres frente a género.

\section{2 - Aspectos de la Historia de las mujeres y género en Argentina}

La socióloga e historiadora Dora Barrancos (2005), en un trabajo de revisión crítica, coloca como antecedentes de la Historia de las mujeres en Argentina a los primeros estudios sobre mujeres que se insertaron en espacios fuera del ámbito doméstico, trabajos no necesariamente ligados orgánicamente al movimiento feminista; luego otros estudios que incorporaron el mundo laboral, la política o el propio movimiento feminista durante el siglo xx; más tarde se agregaron los primeros trabajos sobre siglo xIx y sobre la etapa de la conquista. Pero, más allá de esos antecedentes, es en la década de 1980, con la reapertura democrática, cuando Barrancos considera que se habilitó el crecimiento de una Historia de las mujeres local y hasta regional. En este sentido, afirma: 
[...] las condiciones de posibilidad de una historiografía que se centrara en la condición femenina fueron en la Argentina estas dos vertientes suscitadoras de nuevos problemas, una representada por la crisis de la Historia Social con la adopción -a través de motivos que debitaban al síndrome llamado posmodernismo-, de objetos propios de la Historia Cultural, y la otra representada por las proyecciones del feminismo y de su vigorosa producción intelectual entre las décadas 1970 a 1990 que alcanzaron de lleno a la Historia y conformaron la delimitación de un campo con nombre propio (2005: 54).

En coincidencia con lo expresado por Barrancos, Adriana Valobra y Jorge Nállim (20I6), también en un trabajo de revisión crítica, agregan que en la construcción de la Historia de las mujeres en Argentina:

[...] si bien no estuvieron ausentes las feministas en ese impulso, no puede dejar de reconocerse que también contribuyeron académicas y militantes de partidos de izquierda y otras mujeres que, sin militancia, provenían del campo más estrictamente académico vinculado a la historia social, económica, etc. (2016: 146).

En la década de 1990 se produce un crecimiento exponencial de este campo de estudios, con encuentros académicos sistemáticos, revistas especializadas y una multiplicación de trabajos y temáticas abordadas (Barrancos, 2005). A su vez, Barrancos afirma que los vínculos entre militancia política feminista y desarrollo del conocimiento sobre la temática en el ámbito académico se estrecharon.

La centralidad del concepto de género se da también en esta década. Aunque la autora especifica:

Emergido con cierta timidez en la década de 1970 en el campo de la sociología y de la antropología feminista, la noción fue ganando consideración e introducido de modo precursor en los exámenes de Nathalie Zemon Davies y de Joan Kelly en esa misma década. Y aunque su empleo fue creciente, y contaminó una alta proporción de enfo- 
ques en los países anglosajones, solo bastante más tarde fue recogido en nuestro país. Para evidenciar esa demora basta señalar que en I99I a propósito de las Primeras Jornadas de Historia de las Mujeres desarrolladas en la Universidad de Luján, no apareció «género» como nervadura central de las comunicaciones (Barrancos, 2005: 54).

Algunas de las discusiones que marca Scott en Estados Unidos también aparecen en el ámbito local. En particular, la discusión respecto del esencialismo del concepto de mujer y la ruptura con éste que posibilita la concepción relacional (y también performática) del concepto de género. También el conflicto entre teoría y práctica política es tema de discusión.

A mediados de la década del 2000, Barrancos resume «los tópicos aglutinadores de esta singular empresa colectiva [la Historia de las mujeres y de género en Argentina] fueron "Encierros y sujeciones», "Resistencias y luchas", "Cuerpos y sexualidad”» (2005: 63), considerando además que se trata de "una Historia incipiente que funge todavía como una colcha de retazos» (2005: 65).

\section{$2.3 \cdot$ Musicología y género ${ }^{4}$}

$\mathrm{Al}$ igual que lo sucedido con la Historia de las mujeres para la historiografía, es durante los años setenta, aunque más fuertemente durante los ochenta, que se multiplican los estudios direccionados hacia una historia de las mujeres en la música. En principio como «historia compensatoria", relevando producciones musicales de mujeres, ligas de compositoras, sellos discográficos específicos, etc. El siguiente paso fueron los trabajos que incorporaron perspectivas feministas y, en términos más amplios, de estudios de género (Piñero Gil, 2003). En esta línea se inscriben los libros pioneros de Susan McClary (1991), Feminine Endings:

4 Para esta sección tomamos como referencia central los textos de Piñero Gil (2003), de Cook (2001) y de Ramos (2003), quienes además de aportar elementos de reflexión hacen una revisión de los trabajos musicológicos más influyentes en los estudios sobre mujeres y música así como sobre perspectivas feministas en el estudio de músicas. 


\section{Music, Gender, and Sexuality, y de Marcia Citron (1993), Gender and the musical canon, en Estados Unidos.}

Los trabajos de estas dos académicas estadounidenses formaron parte de lo que se conoce como «Nueva musicología» o «Musicología crítica» (iniciada, entre otros, por Joseph Kerman a mediados de la década de 1980), en la que los estudios sobre música se acercaron a las ciencias sociales y, en particular, a la historia en sus vertientes de historia social y cultural, distanciándose de la tradición positivista imperante en la disciplina ${ }^{5}$.

En este sentido, al cumplirse 20 años de la publicación de su libro, McClary reflexionaba sobre cuál fue su contribución:

\section{[...] respondiendo en parte al extraordinario trabajo de crítica cultural} que se realiza en otros campos, nos esforzamos por llevar ese mismo tipo de preguntas al estudio de la música. Si el género y la sexualidad sirvieron como la lente particular a través de la cual vi la música en Feminine Endings, también traté ampliamente en el libro con muchos otros marcos -colonialismo, raza, psicología, clase social, jerarquía cultural- dentro de los cuales se podría comenzar a desalojar la prohibición de interpretar la música. En otras palabras, yo diría que la principal contribución del libro fue metodológica» (McClary, 20II: 6) ${ }^{6}$.

Se trata de un trabajo que relacionó la musicología con las discusiones contemporáneas en las disciplinas centrales de las ciencias sociales, desde antropología, sociología e historia hasta estudios culturales, crítica de cine y crítica literaria ${ }^{7}$. McClary indica que además de género y

5 La musicología tradicional, imperante hasta los años ochenta, surge de la teoría y la estética musical decimonónica. En clave positivista se dedicó a la construcción de los grandes "genios" de la música (con Beethoven como figura clave), el canon musical clásico, la periodización con explicaciones teleológicas de la historia de la música. Una de las contribuciones del libro de McClary, además del tema central, es poner en la misma bandeja músicas clásicas, pop, música experimental. En este sentido es parte de la "Nueva musicología».

6 La traducción pertenece al autor del artículo. Original: «[...] responding in part to the extraordinary work in cultural criticism occurring in other fields, we ished to bring those same kinds of questions to the study of music. If gender and sexuality served as the particular lens through which I viewed music in Feminine Endings, I also dealt extensively in the book with many other frameworks -colonialism, race, psychology, social class, cultural hierarchy-within which one might begin to dislodge the prohibition against interpreting music. I would claim, in other words, that the book's principal contribution was methodological (McClary, 2011: 6).

7 En la bibliografía de su libro pueden encontrarse referencias a autores tan diversos como Foucault, Adorno, Williams, Hall, Barthes, Geertz o Bourdieu. Del ámbito de la historia de las mujeres toma trabajos de Joan Kelly (Women, 
sexualidad considera otras categorías, claves en las discusiones de esos años, colocándose de este modo en una clara renovación metodológica y teórica de la musicología. En una línea de trabajo similar, Marcia Citron participaba del Feminist Reading Group (creado en 1987), dentro del Center for the Study of Cultures (hoy llamado Humanities Research Center), en Rice University (Houston, USA) ${ }^{8}$.

En sus investigaciones relevaron la construcción de estereotipos de «lo femenino» $y$ «lo masculino» en la narrativa musical (y hasta en la teoría musical), la asignación del ámbito privado y doméstico como primordial para la mujer que tiene como contracara su exclusión de los ámbitos públicos, la construcción y reproducción de canon que configuran las grandes referencias en músicos hombres, blancos, europeos y de clases medias-altas. Procesos que llevaron a exclusión y auto-exclusión de tareas creativas, dificultad en el acceso a formación y al trabajo remunerado, entre otros puntos. Ambas trabajaron con la categoría de género como articuladora de su propuesta.

Poco tiempo después se edita el volumen colectivo Queering the pitch: the new gay and lesbian musicology, con el cual se instala la llamada musicología gay y lesbiana. Se trata de un conjunto de trabajos con metodologías y apuestas diversas que como afirma Brett, uno de los editores, se aglutinan alrededor del interés "por la relación de la música con la identidad, la experiencia y el cuerpo" (Ramos, 2003: 25) ${ }^{9}$. De este libro participa también McClary, por lo cual se puede pensar como ampliación y continuación de la musicología feminista previa.

A diez años de aquellos libros centrales, Pilar Ramos (2003) afirma que:

La tardía incorporación del feminismo a la musicología supone la enorme desventaja de no contar con una tradición y un conjunto de

History, and Theory. Chicago: University of Chicago Press, 1984) y de Denise Riley ("Am I That Name?": Feminism and the Category of "Women" in History. Minneapolis: University of Minnesota Press, 1988).

8 Sus trabajos tuvieron abundantes y enconados detractores, en particular en la American Musicological Society, la institución central de la disciplina en Estados Unidos. En su reflexión luego de 20 años McClary (2011) da cuenta de eso.

9 Aquí puede observarse la incorporación de las perspectivas abiertas por Judith Butler, las cuales McClary no había incorporado en 1991. 
estudios sobre las mujeres comparable al de otras disciplinas como la literatura o la historia general. Pero, al mismo tiempo, esta juventud ha provocado que, casi desde su nacimiento, la musicología feminista esté impregnada del pensamiento posmoderno y viva en estrecha cohabitación con la heterogénea corriente que se ha dado en llamar «nueva musicología». Creo que esto último es, en general, una ventaja, pues permite salvar el aislamiento al que tiende la musicología, quizás, como se ha dicho tantas veces, por lo codificado de la escritura musical (2003: 26).

\section{4 $\cdot$ Musicología y perspectiva de género en Argentina}

Como relata Juan Pablo González (2013), en América Latina -y en Argentina en particular- la musicología se conformó en la primera mitad del siglo xx sobre un ideario nacionalista. Sus principales objetos de estudio fueron las músicas de tradición clásica (de estéticas nacionalistas) y las músicas folklóricas.

Quienes abordaron otros objetos de estudio abrieron el camino para salirse de la lógica nacionalista. Fundamentalmente, desde la etnomusicología y los estudios de la música del período colonial trascendieron las fronteras nacionales, abonando una escucha americanista ${ }^{10}$. Luego, musicólogos estadounidenses, en su proyecto interamericanista, aportaron estudios comparados sobre músicas académicas, coloniales y precolombinas de la región. Un tiempo después, la renovación de la musicología estadounidense comenzada en los 1980 se incorporó también a los estudios sobre música latinoamericana desde el norte. En la propia región esa renovación de la disciplina fue un poco más tardía y tuvo a

10 González (2013) afirma que hay al menos «tres formas principales de orientar la escucha latinoamericana de la musicología en los últimos ochenta años. Estos corresponden al americanismo -surgido en América Latina-, al interamericanismo -impulsado desde Estados Unidos- y a los estudios latinoamericanos -desarrollados en ambas regiones" (2013: 23). A esto suma una cuarta forma, que nota incipiente al momento en que escribe, que corresponde al iberoamericanismo. 
los estudios de músicas populares (consolidados entre los ańos I980 y los 1990) como punta de lanza ${ }^{11}$.

En su libro, Pilar Ramos (2003) da cuenta de que en la música la incorporación de estudios sobre mujeres y de estudios con perspectiva de género es aún más tardía. Graciela Paraskevaídis escribe un artículo pionero en 1986 sobre mujeres compositoras. Pero se destaca por su aporte a una historia de las mujeres en la música el libro de Ana Lucía Frega, titulado Mujeres de la música. Mujeres argentinas (1994), en el que incluye «breves biografías no solo de compositoras, cantantes e instrumentistas, sino también de promotoras y educadoras» (Ramos, 2003: 32). Como afirma Ramos (2003), al igual que la mayoría de los trabajos en castellano hasta comienzos de los 2000 , se trata de trabajos asociados a una primera fase de la musicología feminista que «se caracterizó por centrarse en la búsqueda de datos sobre mujeres sin replantearse las categorías historiográficas» (2003: 32). La recepción de los aportes de la «Nueva musicología» se dan durante las décadas de 1990 y 2000, incluyendo recién allí los aportes de McClary, Citron y sus colegas estadounidenses.

Para los estudios con perspectiva de género en la musicología argentina es una marca de inicio el artículo de Melanie Plesch titulado «De mozas donosas y gauchos matreros. Música, género y nación en la obra temprana de Alberto Ginastera» (2002). Allí la autora expuso los más recientes aportes de la musicología feminista y los aplicó a su estudio de caso $^{12}$. Si bien Plesch no continuó esta línea de trabajo, su posición central en la musicología local favoreció la difusión de este artículo. Luego se destacaron: el trabajo de la española Pilar Ramos (2003) -que desarrolla en extenso el tema por primera vez en castellano-, la traducción del libro de Cook (200I) -que incluye un capítulo sobre el tema-, el número 7 de Dossiers Feministes (2003) -en donde encontramos los trabajos de Piñero Gil (2003) y de Viñuela Suárez (2003) que todavía hoy son referencias centrales-, entre otras bibliografías.

11 El concepto de Música Popular Urbana se constituyó como central en estos estudios, y también la atención de temas de identidad y territorialidades local/global/translocal.

12 Antes presentó una versión más corta de este trabajo en las VI Jornadas de Historia de las Mujeres y I Congreso Iberoamericano de Estudios de las Mujeres y de Género (Buenos Aires, 2001). 
A diez años del trabajo de Plesch, y refiriéndose a las líneas abiertas por McClary y sus colegas, Romina Dezillio (20I2) afirma que:

Este desarrollo no tiene, sin embargo, antecedentes comparables en nuestro país, en lo que se refiere a los estudios sobre música llamada culta o académica ${ }^{13}$. Los trabajos sobre mujeres no cuentan con tradición hermenéutica, quizá porque tampoco se ha dedicado esfuerzo suficiente, todavía, a la sistematización heurística. Por otra parte, los estudios de la musicología norteamericana, si bien proveen un vasto estado de la cuestión y un seductor abanico de perspectivas, están ya de por sí situados según las experiencias de sus autores y en una serie de debates de un campo musicológico que no es el nuestro. Tal situación obstaculiza (o al menos no promueve) un uso contextualizado de las teorías feministas y adecuado al panorama de la musicología local. Por estos motivos, considero elocuente partir de los postulados epistemológicos alternativos (y renovadores) de las teorías feministas, con el objetivo de proponer un uso regionalizado y pertinente a nuestras problemáticas (2OI2: 2I).

A partir de esta propuesta, y fundamentalmente a partir de la renovada presencia pública del feminismo en Argentina y la región en los últimos años, han aparecido trabajos que se hacen eco de este desafío. ${ }^{14} \mathrm{La}$ más reciente muestra de esto es la conformación del colectivo «Músicas y género. Grupo de Estudios Latinoamericano» (MyGLA), creado a comienzos de 2019 e integrado por académicas de Argentina, Chile, Brasil, Colombia y Cuba. ${ }^{15}$ Este trabajo procura sumarse a esa tendencia.

13 Agregaría que tampoco respecto de las músicas populares, al menos en el momento que ella escribía. El sesgo por la música académica (clásica) tiene que ver con su tema de estudio.

14 Son especialmente relevantes las Actas del III Coloquio de Ibermúsicas sobre investigación musical, 'Música y mujer en Iberoamérica: haciendo música desde la condición de género', editado por Juan Pablo González (2017), y el primer número de la revista Contrapulso titulado «Música, género y sexualidad» (2019).

15 A modo de reconocimiento, vale aclarar que el acceso a la musicología feminista, la estadounidense y española pero sobre todo la argentina, es aún más reciente en la UNQ (en donde trabajo). Recién en 2019 se incorporaron Silvia Lobato y Mariana Signorelli al grupo de investigación TeMAC dirigido por Martín Liut, quienes aportaron estas perspectivas. Ellas son parte también del colectivo "Músicas y género. Grupo de Estudios Latinoamericano". 


\section{$3 \cdot$ El DisCo MUJER/MUJER (I984) DE CHANY SUÁREZ ${ }^{16}$}

\section{$3.1 \cdot$ Trayectoria y posición de Chany Suárez}

En esta segunda parte del artículo proponemos un análisis del disco mujer/MUJER (1984) de Chany Suárez. Partimos de preguntas que surgen de lo relatado hasta aquí: dónde estaba, qué lugar ocupaba, qué hizo, cómo fue la trayectoria de Chany, cómo se construye la categoría de mujer en su producción. Tomamos en cuenta, por un lado, las experiencias diversas y a la vez relacionales de género, y, por otro lado, aspectos de cómo se consideraba socialmente a las mujeres, en particular en las músicas populares (Viñuela Suárez, 2003; Patiño Mayer, 20I7; Adorni, 2019; Valdebenito, 2020).

En un trabajo anterior (Mariani, 2019a) ubicamos a Chany Suárez ${ }^{17}$ como una de las artistas emergentes del folklore argentino hacia el fin de la dictadura. Retomamos algunos puntos que nos son necesarios para pensar el disco de 1984 .

Chany nació en Capitán Sarmiento, a $145 \mathrm{~km}$ de la ciudad de Buenos Aires, y se crió en Don Bosco (partido de Quilmes), en el conurbano bonaerense. A fines de la década de 1960 comenzó su carrera como cantante junto a su hermano Julio Lacarra ${ }^{18}$. Con él conocieron en Cosquín (provincia de Córdoba) a Armando Tejada Gómez y a César Isella, quienes la convocaron para grabar una zamba en 1971. Esa fue su primera participación en un fonograma. En 1974 editó su primer disco, Me gusta andar, en donde se perfiló tanto el sonido como el tipo de repertorio que abordó en los siguientes años. Incluyó clásicos del folklore de referentes del Movimiento del Nuevo Cancionero (MNC) y relacionados al grupo literario La Carpa, de compositores nuevos y de otros géneros populares (principalmente latinoamericanos). Los arreglos estuvieron a cargo

16 Una versión previa de esta sección fue presentada en la "Jornada de Intercambio de Trabajos Musicológicos y Conversatorio: Musicología, Investigación y Género" realizada el 4 de noviembre de 2019 en la Universidad Nacional de Quilmes (coorganizada por TeMAC, MyGLA y la Cátedra Abierta de Géneros y Sexualidades UNQ). Agradezco los comentarios recibidos, algunos de los cuales incluí en este artículo.

17 Elvira Susana Suárez (1950).

18 Julio César Suárez (1947). 
del guitarrista Daniel Homer (1948), quien además era pareja de Suárez. Desde ese primer disco trabajaron con una amplia hibridación de géneros musicales. Homer y Suárez estaban en relación estrecha con músicos del rock como Litto Nebbia y de la escena de la proyección folklórica como Manolo Juárez, así como con la escena cordobesa de donde es oriundo Homer (Mariani, 20I9a: I82).

Con estos primeros datos podemos relevar que Chany construye su carrera artística rodeada de referentes hombres: su hermano y su pareja, Tejada Gómez e Isella, Nebbia y Juárez, y también los integrantes de su grupo. Luego Gustavo "Cuchi» Leguizamón, con quien comienzan una relación artística a partir de que ella graba Zamba del laurel en 1980. Esto tiene al menos dos caras: una de condicionamiento y limitación de lo posible como mujer (y como joven, ya que son todos hombres mayores que ella en edad), y otra como legitimación de su tarea artística frente a los estigmas de la mujer "pública» ${ }^{19}$.

También su repertorio está constituido principalmente por composiciones de hombres (si bien aparecen algunos centrales para el canon folklórico de la época, también incluye otros menos conocidos). Aunque, para ese entonces, incluyó letras de Mirtha Defilpo ${ }^{20}$ (de allí toma el título para el álbum) y Marta Lynch en su segundo disco (1976), de Corina Vilella y de la propia Chany en su disco de música infantil (1979), en donde Chany también compone la música de un texto suyo, y en su disco Abierto al júbilo (1980) vuelve a aparecer Defilpo y Chany aporta un texto y una canción con letra y música.

La interpretación, especialmente de la voz, resulta ser el espacio en el que es más aceptada y reconocida la participación de las mujeres en la música ${ }^{21}$. Es así que la mayor cantidad de referencias con que contaba

19 Quizás en este sentido puede leerse también el uso de vestimenta generalmente formal por parte de Chany, relacionable con mujeres mayores que ella. Tanto la vestimenta como el apoyo explícito de los hombres que rodean a una mujer artista resultan estrategias muy recurrentes de legitimación de éstas. El estudio de Angélica Adorni (2019) sobre el caso de Ramona Galarza es muy claro en ese sentido, así como el de Patiño Meyer (2017) sobre Ángela Irene y Teresa Parodi, y funcionan como antecedente en el campo del folklore.

20 Defilpo, que aporta además un texto en la contratapa del disco, resulta ser una par directa de Chany por edad y por trayectoria. También ella se encontraba desarrollando su carrera artística en un ámbito predominantemente masculino (vale recordar que era pareja de Nebbia en ese entonces).

21 Viñuela Suárez (2003) desarrolla muy claramente esto en músicas populares urbanas. 
Suárez eran intérpretes. La más reconocida es sin dudas Mercedes Sosa con quien mantenía una relación artística en esos años (participó en los conciertos de la vuelta del exilio). Una de las excepciones más sobresalientes es María Elena Walsh, reconocida compositora además de intérprete. Y es justamente en el disco de música infantil, en la que Walsh se destaca, donde Chany incluye por primera vez una composición propia.

El disco No te rindas (1982), grabado luego de la Guerra de Malvinas, fue con el que Suárez logró mayor difusión. En ese álbum incluyó dos canciones que resultan ser relevantes para pensar la construcción de Suárez como enunciadora respecto de la categoría «mujer». Una es la canción de Silvio Rodríguez titulada Mujeres. En ella se hace una valoración de las mujeres -conocidas y desconocidas- aunque reafirmando algunos lugares tradicionales ${ }^{22}$. La otra es No te rindas, Malena de Roque Narvaja. Esta canción trata las dificultades de una mujer adolescente frente a distintos mandatos sociales, con énfasis en las presiones que sufre por ser mujer y joven. Frente a esto, el estribillo de la canción la alienta diciéndole «no te rindas».

Vemos entonces que Chany desarrolla su trayectoria en un ambiente predominantemente masculino, a pesar de lo cual incluye referentes mujeres, incursiona en la composición e incorpora canciones que hablan específicamente sobre las mujeres, reivindicándolas y contando algunas de sus dificultades en la vida social.

\section{$3.2 \cdot$ El disco mujer/MUJER (I984)}

Para el análisis que sigue tomamos en cuenta aspectos de la tapa, contratapa e interior del álbum, el título, el repertorio, sus letras y músicas.

\footnotetext{
22 Lorena Valdebenito (2020) detalla, en su trabajo sobre la Nueva Trova Cubana, sobre esta canción: “'Me estremeció la mujer' Ideario de diferentes mujeres (la mujer madre, la mujer del poeta, la mujer joven, los ojos de su hija, su abuela... un montón mujeres)" (2020: 138). Y en una lectura más panorámica de Rodríguez, afirma: "La figura de la mujer en las canciones de Silvio Rodríguez es imaginada y representada como un objeto simbólico de lo femenino fijo e idealizado desde de los códigos heteronormativos, pero dentro de ese marco fijo, es representada de diferentes formas, pues adquiere múltiples significaciones. A pesar de ello, es posible notar que en la mayoría de las canciones se produce un diálogo binario que transita entre dos espacios: lo político y el amor, habitualmente desde la lírica en un plano metafórico" (2020: 156).
} 
Esos aspectos toman relevancia como parte del disco en tanto dispositivo de enunciación dentro de una red de relaciones y en el marco de la trayectoria de Suárez.

Según relata la propia Suárez en su página $\mathrm{Web}^{23}$ :

En la tapa de este álbum titulado mujer / MUJER, hay dos fotos de Chany: una tomada en su primera infancia y la otra en 1984, el año en que lo grabó. Las grafías en minúscula y mayúscula, remedan intencionalmente las fotos como una metáfora que nos conduce desde el principio a una perspectiva. Entre esas dos mujeres que ha sido Chany, hay una carrera artística que no se detuvo y un testimonio de esa trayectoria a través de varios discos (Suárez, s/f).

Con esta intervención sitúa el título tanto en su historia personal como en su crecimiento artístico, aunque no en el de «la mujer» en un sentido colectivo más amplio. En la sección de recortes de prensa de su página Web incluye una nota de época, al momento de salir el disco, donde afirma que el título «significa la mujer hecha, transcurrida ya la etapa de crecimiento» (Suárez, s/f). En la misma nota, en un recuadro resaltado, afirma: «cuando firmé mi primer contrato, me dijeron que las mujeres no venden discos. Ahora, las mejores ventas corresponden a las mujeres. Parece que las cosas cambiaron» (Suárez, s/f). Con este último comentario sí posiciona el disco dentro de una disputa de «las mujeres». La trayectoria es personal pero también es colectiva. Hay una percepción en Suárez de que las condiciones de producción, para ella y para «las mujeres», han cambiado. Se han alcanzado derechos, se han logrado ocupar espacios públicos, por lo cual es posible ponderar la trayectoria.

Otro aspecto del título que va en la misma dirección es la repetición. No solo es la palabra mujer primero en minúsculas y después en mayúsculas, sino que la propia repetición de la palabra funciona como resaltado. Es una mujer completa, «la mujer hecha, transcurrida ya la etapa de crecimiento» (Suárez, s/f) ${ }^{24}$.

23 En la sección tapas y comentarios de cds.

24 Agradezco a Silvia Lobato la observación de este punto. 
El sonido del disco sigue la línea del anterior ${ }^{25}$. Además de la voz de Chany, los músicos que participan son Daniel Homer en guitarras y arreglos, Enrique "Zurdo» Roizner en batería, Jorge Rabito en bajo, Mingui Ingaramo en piano y Obi Homer en quena. Aquí se repite lo que relevamos más arriba: son todos músicos hombres. Chany ocupa el lugar central como intérprete vocal pero dejando el lugar legitimado como creativo en Homer, quien hace los arreglos, y el lugar del virtuosismo instrumental también repartido entre "los músicos» (entre los cuales sobresale Homer y sus guitarras en el sonido de todo el disco).

La elección del repertorio resulta ser central para quienes son intérpretes en las músicas populares. Allí se suelen ejercer opciones estratégicas claves. En el folklore esto es bien claro en figuras centrales como Jorge Cafrune o Los Chalchaleros, por ejemplo. Para nuestro caso es especialmente importante la figura de Mercedes Sosa, quien desde el comienzo de su carrera fue delineando los ideales del Movimiento del Nuevo Cancionero (MNC) con estrategias contundentes en la elección del repertorio. En 1982, al volver a Argentina, en los conciertos de febrero y en el disco que editó luego a partir de ellos, llegó a incluir géneros como el rock nacional pasando por canciones latinoamericanas (de Violeta Parra a la Nueva Trova Cubana), tango y canciones de distintos sectores del folklore argentino. Por ocupar un lugar central en el folklore y en las músicas populares argentinas del momento, Mercedes habilita y legitima ese tipo de elecciones en artistas emergentes.

En el caso de Chany Suárez ya desde su primer disco tomó canciones del folklore de distintos sectores, canciones latinoamericanas, del rock local, del pop. Desde ese entonces suele combinar la elección de temas de artistas reconocidos del folklore con otros emergentes y de otros géneros populares. En esa misma línea construye el repertorio de mujer/MUJER.

Nos detenemos a continuación en aspectos de las canciones que consideramos relevantes en la construcción del dispositivo de enuncia-

25 Algunas de las características son las que nombramos en esta cita: «El sonido 'limpio’, nítido, con peso en los agudos y a la vez con procesamientos (reverberación, 'delay', 'chorus', etc.) de todos los instrumentos, pero especialmente de las guitarras de Homer (muy influido por Pat Metheny y el jazz fusión de la época), aleja el sonido del grupo tanto del folklore como del rock. La voz de Suárez toma usos propios del folklore del noroeste (el típico quiebre de la voz), del tango y también del pop" (Mariani, 2019a: 184). 
ción. Si bien prestamos más atención a las relaciones con el título pretendemos dar cuenta de la complejidad del enunciado en su conjunto.

El disco comienza con Levantate y canta de César Isella con letra de Héctor Negro. Isella grabó esta canción en 1983 en su disco Para volver cantando, ubicada también como primera pista. Con el ex integrante del grupo Los Fronterizos y figura central del MNC tenían una relación de más de diez años, pero se agrega en este caso que prácticamente todo el grupo de Chany toca en su disco. Además, Daniel Homer hace los arreglos. De este modo, Isella como figura consagrada del folklore renueva el sonido de su propuesta y legitima esta línea de producción. Suárez a su vez reafirma su vínculo con figuras del MNC y con un ideario enfrentado a la dictadura. La canción no es estrictamente del folklore sino que se acerca al cancionero más melódico del tango ${ }^{26}$, con una letra que afirma la esperanza frente al dolor y a un golpe «a contrapelo, a contrasol, a contraluz, a contravida».

La segunda canción es La mala gente de Amaury Pérez, un referente de la Nueva Trova Cubana (NTC) ${ }^{27}$. Con esta elección Chany amplía sus referencias en ese movimiento cubano más allá de las figuras centrales ${ }^{28}$.

La tercera pista es Coraje mujer de Vitor Martins e Ivan Lins. Se trata de la primera canción que establece un vínculo directo con el título del álbum, al menos por contener la palabra mujer. El brasileño Lins, referente de la Música Popular Brasilera (М в ) y relacionado tanto al rock como al pop, empezaba a ser reconocido en Argentina en esta etapa (unos meses después grabó un disco en vivo en el Luna Park con Pedro Aznar, León Gieco y Luis Alberto Spinetta como invitados ${ }^{29}$ ). Se trata otra vez de una elección que amplía los vínculos a músicas latinoamericanas desde una propuesta abierta no solo al rock sino también al pop. De todos modos, no es menor en la elección de este autor y esta can-

26 En el arreglo de Isella esto es más claro al incluirse el bandoneón de Daniel Binelli en la instrumentación.

27 A partir de aquí abreviamos NTC (Nueva Trova Cubana).

28 Como afirmamos anteriormente, Chany había grabado para ese entonces varias canciones de Silvio Rodríguez. Este último junto con Pablo Milanés eran las figuras más reconocidas de la NTC en la Argentina de esos años (como muestra de eso, en 1984 dieron conciertos juntos en el Estadio Obras Sanitarias).

29 Se trata del concierto de Ivan Lins en el Luna Park el 7 de septiembre de 1984, que se editó en LP con el nombre Encuentro (1984). Allí grabó en vivo también Coragem, mulher, pero antes la había incluido en versión de estudio en el álbum Novo Tempo (1980). 
ción, la complejidad armónica y melódica que presenta, siendo esta una de las marcas sobresalientes de la propuesta de Chany y de su guitarrista y arreglador Daniel Homer.

La mujer que describe la canción se caracteriza por el coraje, la firmeza y lo delicado, la certeza y la congoja, lo suave y lo fuerte, la energía, la virtud, la fe, la templanza, la petulancia, la decencia, la ternura, la paciencia, el misterio y el encanto. Son una serie de características que le permiten «seguir de pie hasta el fin». No se describen obstáculos pero se entiende que si esas son las virtudes es porque los enfrenta. Aquí se mezclan aspectos de una persona activa con otros de una persona pasiva frente a conflictos o desafíos. El momento que sitúa más claramente en un lugar pasivo a la persona que describe es cuando dice «¿es que hay un guía que te alumbra cada día?». En ese caso sería alguien externo (¿Dios?) y masculino quien está promocionando esa mujer virtuosa. Pero más allá de ese momento, la letra apunta a relevar una mujer con coraje como ideal de mujer.

Al ser cantada por Chany en este disco llamado mujer/MUJER funciona como una autoafirmación. Al igual que en la canción no se explicitan cuales serían los conflictos o dificultades que enfrenta, no hay una problematización clara de eso, pero sí hay una intención de fortalecimiento como enunciadora: una enunciadora con coraje, que se posiciona como mujer en lo personal y en lo artístico. Retomando la presentación que hace años después en su página Web, que se condice con la serie de fotografías que incluye en la tapa y en el interior del $\mathrm{LP}^{30}$, se trata de valorar su crecimiento: ya no es una nińa o una artista que recién comienza sino una mujer con mayúsculas y una artista con trayectoria.

La cuarta canción es Anillo chapaco, un huayno de César Isella con texto del poeta boliviano Octavio Campero Echazú. Se trata de una canción que, con referencias a la región de Tarija -chapaco es un vocablo que hace referencia a quien habita esa zona-, habla del anillo que el personaje de la letra hace fabricar para unirse a la mujer que ama. El anillo, según afirma, «ella lo quiere más firme que la muerte y el olvido» y le pide al

30 En el interior del LP se incluyen fotos de Chany, ordenadas cronológicamente, desde muy pequeña hasta las primeras actuaciones profesionales. 
platero que así lo haga mientras «ella me espera lavando su ropa blanca en el río». Más allá del "cariño» con que lo expresa se trata de una afirmación de roles tradicionales con el hombre decidiendo, hablando por la mujer y colocándola en un lugar pasivo, de espera.

La quinta canción y cierre de la faz a del disco es Conciencia y corazón con letra de Chany y música de Juan Carlos Ingaramo, amigo de Suárez y Homer además de referente emergente de la música cordobesa en estos ańos. Sobre una rítmica cercana al chamamé se arregla una balada pop que, como una afirmación moral, opone la dupla conciencia y corazón como virtudes, frente a la oscuridad y la maldad. En la faz B como octava pista se incluye Guiño de ilusión también con letra de Suárez y con música de Mingo Ingaramo (hermano de Juan Carlos) y Daniel Homer. La letra enfrenta la nińez y la adultez como espacios de ilusión y de realidad respectivamente. El personaje de la letra, que no tiene género definido, recorre la nińez sin temor y como un juego (el carrusel), mientras la adultez es con temor y con cuentas que pagar. En esta balada pop lenta Chany se identifica nuevamente con el crecimiento y la madurez, en línea con el título del disco, la serie de fotografías y la explicación hecha en su página Web.

En las dos letras escritas por Chany se evitan las identificaciones de género. También ocurre lo mismo en otras canciones del repertorio (Levantate y canta, La mala gente, Palabras autorizadas). En un contexto de canciones escritas por hombres, con un grupo compuesto por hombres y una trayectoria rodeada de hombres, es sin dudas una estrategia para posicionarse como enunciadora. No desde una denuncia, rechazo o problematización de otras posiciones sino desde una ampliación de lo posible como «mujer/MUJER» ${ }^{31}$.

La faz в del LP cuenta con tres canciones de Julio Lacarra ${ }^{32}$, hermano de Chany. La que ocupa el sexto lugar es Cada vuelta que doy, con

31 Como comentamos, Scott (2003) releva que, en el ámbito de la historia en Estados Unidos, no discutir la objetividad de las historias tradicionales y construir relatos paralelos fue un recurso táctico que permitió lograr un lugar y validación propio para la historia de las mujeres. Puede pensarse de un modo similar este tipo de opciones tomadas por Chany: afirma su lugar sin recurrir a confrontaciones.

32 Es un porcentaje alto del repertorio, 3 de 10. Lo mismo sucede en otros discos de Chany (anteriores y posteriores). Esto realza la importancia de su hermano en su trayectoria y posicionamiento. 
texto de Hamlet Lima Quintana. Sobre un ritmo cercano al vals peruano el personaje de la letra elije la alegría para enfrentar el sufrimiento. La novena canción es Palabras autorizadas, un chamamé que se sitúa en los trenes, que bien podrían ser los que van de Don Bosco a Constitución (trayecto que sin dudas habrán hecho tanto Julio como Chany), oponiendo esas personas que viajan con sus urgencias y dramas frente a flacas promesas en frases ilustradas. Y la décima canción es La canción de nuestros días, que Lacarra grabó el año anterior en su disco homónimo y funciona como un manifiesto en el fin de la dictadura: «No me voy de aquí porque es mi tierra y soy algo dura de sesera, esta es la que piso y me sustenta quiero estar cuando apure la primavera» [...] «No me voy de aquí porque a mi gente no quiero llorarla desde afuera, la pena entre muchos duele menos y se hace más aguantadera». En el contexto de un repertorio de canciones compuestas por hombres, salvo sus propias letras que no indican género, en La canción de nuestros días es en la única en la que Chany interviene el texto cambiando la terminación de masculina a femenina en la primera estrofa. De este modo realza la identificación de la cantante con el personaje de la letra y su relato.

La séptima canción es la cueca La arenosa con música de Gustavo Leguizamón y letra de Manuel Castilla, referentes centrales del folklore salteño vinculados al grupo literario La Carpa. Se trata entonces de una adscripción a esa vertiente dentro del folklore y una cita de autoridad. Pero también un intento de afianzar las relaciones con Leguizamón, a quien le había gustado ${ }^{33}$ la versión de Zamba del laurel que Chany grabó en Abierto al júbilo (1980). Dentro de un disco con canciones de diversos géneros y un sonido entre el pop y el jazz fusión ${ }^{34}$, la inclusión de esta cueca tan reconocida y vigente ${ }^{35}$ ancla la propuesta en el campo del folklore. A su vez la versión se construye con el virtuosismo instrumental en primer plano, por lo cual posiciona la propuesta con claras intenciones renovadoras para este ámbito. La voz de Chany trabaja en el mismo

33 Así lo cuenta Chany en varias entrevistas y en su web.

34 Ver nota al pie número 25.

35 Por ejemplo, Mercedes Sosa la incluyó en su disco en vivo al volver del exilio en 1982. 
sentido: amplitud de intensidades y de registro, proyección, agilidad, saltos interválicos amplios, dicción clara. Una voz virtuosa, con coraje.

La elección del repertorio, así como su tratamiento, dan cuenta de un dispositivo de enunciación complejo. Hay varias opciones estratégicas que en línea con el título proponen una «mujer» que crece y excede los espacios, actividades y actitudes más tradicionales. Pero no todas van en ese sentido, también se afirman estereotipos. Otras opciones estratégicas apuntan a una ampliación de vínculos con músicas y referentes de esas músicas (MNC, NTC, MPB, etc), otras a posicionarse en el contexto político (en contra de la dictadura), otras a validarse en términos de virtuosismo instrumental/vocal y complejidad armónica. Estas últimas opciones estratégicas que nombramos también contribuyen a la construcción de una Chany Suárez enunciadora con una trayectoria sólida.

\section{$4 \cdot$ Reflexiones finALES}

El propio enunciado del disco analizado propone la categoría «mujer» como punto central. Abordar el análisis con perspectiva de género nos permitió considerar la construcción de Chany Suárez como enunciadora en relación a esa categoría («mujer»).

En su disco inmediatamente anterior, No te rindas (1982), trató el tema en la canción que da nombre al disco y en Mujeres de Silvio Rodríguez ${ }^{36}$. Luego de esas incursiones, titular su nuevo álbum mujerl $M U J E R$, conteniendo además la canción Coraje, mujer y canciones propias que aluden a su trayectoria, parece retomar con más centralidad la discusión sobre relaciones de género. Las estrategias de construcción de una Chany Suárez enunciadora en este disco apuntan a una afirmación personal y artística, a remarcar la trayectoria y el coraje, y no a una denun-

36 Por supuesto, visto desde hoy, tras varios años de luchas y una alta visibilización de las discusiones, los planteos de estas canciones resultan muy leves y hasta reproductores de una lógica patriarcal, pero para hacer justicia histórica debe considerarse el estado del debate en ese momento. Se registraban algunas tensiones pero los discursos feministas y de género, si bien ya tenían un fuerte desarrollo en algunos sectores, se fueron ampliando en los años siguientes, y se masificaron recién en las primeras décadas del siglo XXI. Ver Barrancos (2005), Andújar (2012), Valobra y Nállim (2016). 
cia, rechazo o problematización de otras posiciones (las de «los hombres», por ejemplo). Por lo cual, se construyen enunciatarias/os que no son necesariamente feministas pero que valoran la ampliación de espacios posibles para las mujeres.

En la selección del repertorio, así como en el arte de tapa e interior del disco y en la propuesta sonora, pueden observarse las tensiones que Chany enfrenta respecto a roles de género. Procura caminos alternativos posibles, se afirma en lo individual y también como parte de un colectivo («las mujeres» artistas, que venden discos). Pero a la vez sostiene los roles tradicionales, no solo del campo del folklore sino también del espacio social general.

Con esas tensiones, el disco, así como la trayectoria de Suárez, sin dudas aportan y forman parte de una ampliación de lo posible para «las mujeres» músicas en esta etapa. Esto puede interpretarse en línea con lo señalado por Viñuela Suárez: «la opción más práctica e interesante para las mujeres en general, y para las músicas en particular, consiste en dirigir los esfuerzos hacia la subversión y la ampliación de la feminidad tradicional» (2003: 22).

La Historia de las mujeres, el feminismo y la categoría de género han descentrado y ampliado tanto los objetos de estudio como las fuentes de información, pero sobre todo han habilitado nuevas preguntas ${ }^{37}$. Han permitido considerar en términos relacionales la construcción y significación de categorías como «mujer»y «hombre». Con la política feminista desde los años sesenta, y en Argentina más fuertemente en los años ochenta con la reapertura democrática, la reflexión sobre las mujeres y más ampliamente las relaciones de género tuvo un notable crecimiento (tanto en la academia como en diversas disciplinas artísticas). Retomando la propuesta de Dezillio (20I2), que citamos más arriba, en este trabajo procuramos situar en un caso local los aportes de la musi-

37 Las que surgen en el relato de la primera parte de este artículo: dónde están/estaban, qué han/habían hecho y cómo se considera/consideraba socialmente a las mujeres, cómo contener las experiencias de ambos sexos de modo relacional en nuestro relato histórico, cómo se construyen las categorías de "mujer» u "hombre" en un momento y lugar dados. En nuestro caso, surgen de allí las preguntas por Chany, su música, el marco de relaciones de género y las tensiones con las que trabajó, así como por la construcción de una enunciadora «mujer» en su disco. 
cología feminista y de género, haciendo pie también en herramientas de la historiografía de las mujeres y de género.

\section{REFERENCIAS BIBLIOGRÁFICAS}

ADORNI, ANGÉLICA (2019): «¿Un hada bienhechora en el baile de sirvientas? Ramona Galarza y sus discos de música litoraleña argentina en los sesenta", en Contrapulso, 1/1, pp. 1-19.

ANDÚJAR, ANDREA (2012): «El género de la Historia. Aportes y desafíos para el estudio del pasado", en VIANO, Cristina (coord.), Miradas sobre la Historia. Fragmentos de un recorrido, Rosario, Prohistoria, pp. 87-102.

BARRANCOS, DORA (2005): "Historia, historiografía y género. Notas para la memoria de sus vínculos en la Argentina", en La Aljaba, pp. 49-72.

BRETT, PHILIP, ELIZABETH WOOD Y GARY THOMAS (ed.) (2006): Queering the pitch: the new gay and lesbian musicology. USA, Taylor \& Francis Group, V.O. 1994.

Citron, MARCia (1993): Gender and the musical canon. Gran Bretaña: Cambridge University Press.

COOK, NiCHOLAS (2001): "Música y género" en De Madonna al canto gregoriano. Una muy breve introducción a la música. Madrid, Alianza Editorial, V.O. 1998.

DEZILlio, Romina (2012): “Historizar la experiencia. Hacia una historia de la creación musical de las mujeres en Buenos Aires (1930-1955): fundamentos, metodología y avances de una investigación", en Boletín de la Asociación Argentina de Musicología, año 27, n68, Córdoba, 18-27.

DÍAZ, CLAUDIO FERNANDO (2009): Variaciones sobre el ser nacional. Una aproximación socio discursiva al folklore argentino. Córdoba, Recovecos.

gonzÁlez, JuAn pablo (2013): Pensar la música desde América Latina, Buenos Aires, Gourmet Musical.

MARIANI, TOMÁs AGUSTÍN (2019a): "De la vuelta de Mercedes Sosa a la asunción de Raúl Alfonsín: actores emergentes en el folklore argentino", en Revista Argentina de Musicología 19 (2018), 175-193.

(2019b): "Folklore y transición (1982-1984): estrategias en torno a la canción ‘Grito santiagueño’ de Raúl Carnota ", en Recial, 10 (16), pp. 206-223.

(2020): «El folklore en el año de la Guerra de Malvinas (1982): el disco Mercedes Sosa en Argentina", en Música e Investigación 27 (2019), pp. 71-93. MCCLARY, SUSAN (2002): Feminine Endings: Music, Gender, and Sexuality, Minnesota, Univiversity Of Minnesota Press, V.O. 1991. 
(2011): "Feminine Endings at Twenty", en TRANS-Revista Transcultural de Música/Transcultural Music Review 15, pp. 1-10.

PATIÑO MAYER, LUCÍA (2017): “Tácticas y recorridos artísticos en el campo del folklore durante la última dictadura militar argentina: dos mujeres y dos canciones", en Actas del X Seminario Internacional Políticas de la Memoria. Arte, política y memoria, Mesa 11, Centro Cultural de la Memoria Haroldo Conti, pp. 1-9.

PIŃERO GIL, CARMEN (2003): "La transgresión de Euterpe: música y género", en Dossiers Feministes, Na 7, pp. 45-63.

PLeSh, MELANIE (2002): "De mozas donosas y gauchos matreros Música, género y nación en la obra temprana de Alberto Ginastera", en HUELLAS...Búsquedas en Artes y Diseño, №2, pp. 24-31.

sCOTT, JOAN (1990): "El género: una categoría útil para el análisis histórico», en James S. Amelang y Mary Nash (eds.), Historia y género. Las mujeres en la Europa moderna y contemporánea. Valencia, Ediciones Alfons el Magnánim. Hay versión del artículo de 1986.

(2003): «Historia de las mujeres", en Peter Burke (ed.), Formas de hacer Historia. Madrid, Alianza. V.O. 1991.

SUÁREZ, CHANY (s/f): Web oficial. https://chany-suarez.webnode.com/ 